\title{
New method for determining apparent axial center of rotation of lumbar and thoracic spine segments
}

\author{
Sanjum P. Samagh, MS; ${ }^{1}$ Charles D. Rosen, MD; ${ }^{1-2}$ Karimdad Otarodifard, MD; ${ }^{1}$ Matthew Kornswiet, MS; ${ }^{1}$ \\ Gabe Palmer, BS; ${ }^{1}$ Thay Q. Lee, PhD $^{\mathbf{1}^{*}}$ \\ ${ }^{1}$ Orthopaedic Biomechanics Laboratory, Department of Veterans Affairs Long Beach Healthcare System, Long Beach, \\ CA; and University of California, Irvine, CA; ${ }^{2}$ Association for Medical Ethics, Manhattan Beach, CA
}

\begin{abstract}
One main question in spinal kinematics is the determination of the spine's apparent axial center of rotation. Previous research on this topic has yielded contradictory results. The objective of this study was to determine the apparent axial center of rotation for seven lumbar and six thoracic spinal segments by developing and validating a new method. A custom six-degree-of-freedom device, allowing full range of motion, was used with motion recording and analysis software. This system tracked a grid of markers on a specimen when rotational torque was applied in both clockwise and counterclockwise directions at $3.53 \mathrm{Nm}, 7.05 \mathrm{Nm}, 10.58 \mathrm{Nm}$, and $14.10 \mathrm{Nm}$. The area encompassing the apparent axial center of rotation was determined by identifying the five markers with the least amount of motion. The marker angular displacement was calculated as the angle between a virtual line drawn between two points in the initial and final torque conditions. Rotation in both directions was averaged. The lumbar and thoracic spinal segments averaged an apparent axial center of rotation at the posterior border of the vertebral endplates and the anterior border of the spinal canal, with average clockwise to counterclockwise angular displacement ratios of 0.87 and 0.97 , respectively.
\end{abstract}

Key words: axial, biomechanics, center of rotation, lumbar spine, new method, rehabilitation, rotational torque, spinal injuries, spinal kinematics, thoracic spine.

\section{INTRODUCTION}

The human spine is an anatomically and functionally complex structure, responding daily to vigorous loading and rotational conditions. In order to appropriately treat pathological spinal injuries and deformities, researchers and clinicians must understand the complex motion of the spine, particularly the spinal center of rotation. Much research has been done regarding measurement of the center of rotation of the spine, but the results are often contradictory, placing the center of rotation anywhere from the center of the vertebral body to inside the spinal canal to the tip of the spinous processes [1-7].

Methods for determining the center of rotation of the spine vary and include in vitro and in vivo studies, radiographic analysis, displacement jigs, and geometrical correlation to determine the apparent axial center of rotation. One of the first groups to determine the center of rotation in in vivo subjects was Gregersen and Lucas in 1967 [8]. Basing their findings on in vivo experiments and Rockwell et al.'s geometric work [9], they concluded that the spinal center of rotation can be determined geometrically as the intersection of two lines passing through the centers of the surface of the articular facets. Nash and Moe's 1969 study took the center of rotation as the line of the

Abbreviations: $\mathrm{DOF}=$ degree of freedom, $\mathrm{L}=$ lumbar, $\mathrm{T}=$ thoracic.

*Address all correspondence to Thay Q. Lee, PhD; Orthopaedic Biomechanics Laboratory, VA Long Beach Healthcare System (09/151), 5901 East 7th Street, Long Beach, CA 90822; 562-826-5344; fax: 562-826-5675.

Email: tqlee@med.va.gov; tqlee@uci.edu

DOI:10.1682/JRRD.2010.09.0168 
posterior longitudinal ligament based on their own experiments [10] and Roaf's theories [11]. Several other authors later developed sophisticated testing apparatuses and techniques, often fixating portions of the spinal segment, which limits physiological motion. In 1984, Panjabi et al. developed a jig that applied flexion and extension forces and located the thoracic $(\mathrm{T})$ center of rotation as below the geometric center of the moving vertebrae [12]. Kotani et al., in determining the effectiveness of retaining motion in current intervertebral disc replacement models, used lateral plain films and superimposed flexion-extension images to determine the center of rotation as the posterior third of the intervertebral disc [13]. Molnár et al. later used several methods, including geometric correlation, volume change, and cadaveric spine testing, to support their hypothesis that placed the T center of rotation in the anterior portion of the spinal canal [14].

The necessity of establishing an accurate center of rotation stems from an attempt to preserve physiological motion in pathological spinal conditions. The purpose of this study is to expand upon the current understanding of spine kinematics. Developing an in vitro device allowing six-degree-of-freedom (DOF) motion may help objectively resolve contradictions in previous research regarding the apparent axial center of rotation. Based on the radiographic analysis of Kotani et al. [13] and summation data from Molnár et al. [14], we hypothesize that the apparent axial center of rotation is located near the posterior border of the vertebral endplates and the anterior border of the spinal canal. According to Molnár et al., the farther the rotational axis is from the center of the spinal canal, the larger the shear forces on the spinal center of rotation [14]. Avoiding shear forces on the spinal cord is instrumental in preserving neurological function; therefore, the apparent center of axial rotation is likely somewhere near the cord running through the spinal canal. Therefore, the objective of this study is to use a custom six-DOF device, which allows for full, free range of motion, to determine the axial center of rotation of the lumbar (L) spinal segments. We focused on the $\mathrm{L}$ spine because this region is frequently associated with degenerative disc diseases, such as anterolisthesis and intervertebral disc herniation [15].

\section{METHODS}

To determine the apparent axial center of rotation, we used a custom six-DOF device and a digital video analysis system (Figure 1). The digital video analysis system included a Canon GL2 camcorder (Canon USA, Inc; Lake Success, New York); WINanalyze software (Mikromak; Berlin, Germany) was used to track markers, placed on the specimen to create a grid, during application of axial torque. WINanalyze captured the motion of the grid markers and exported the $x$ - and $y$-coordinates of the markers, which allowed us to calculate the distance each marker moved. The software captured the motion of each marker every 0.05 seconds to track any irregularities in motion.

Four validation experiments were used to verify the WINanalyze software and the custom testing device.

\section{Validation 1}

The first validation experiment tested whether the WINanalyze software accurately determined the axial

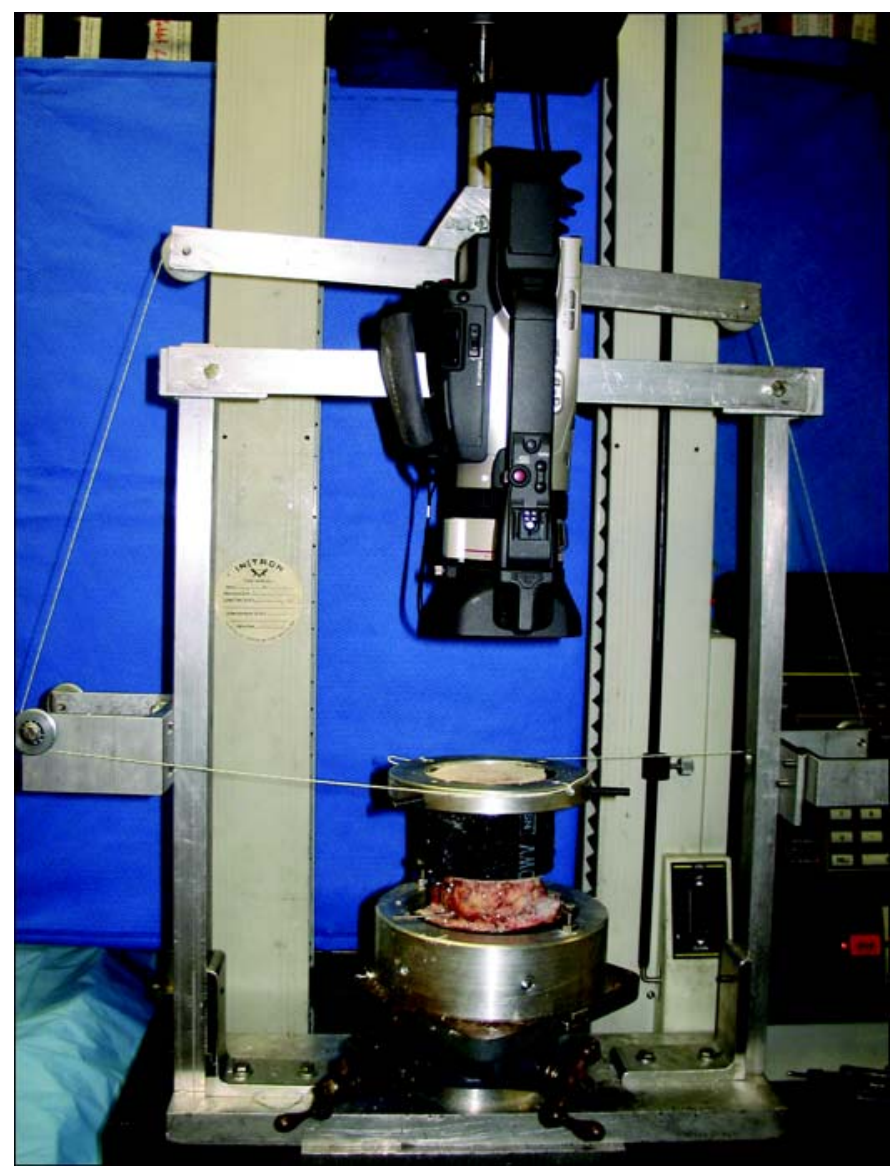

Figure 1.

Custom six-degree-of-freedom device with lumbar segment loaded into base. Fishing wire applies axial rotation onto segment while Instron machine moves upward. Canon GL2 digital video camcorder records movement of segment by tracking markers on plastic grid. 
center of rotation in a known model. The GL2 camcorder and WINanalyze motion software tracked the grid markers' motion in a model with a known center of rotation. This was repeated five times with approximately $20^{\circ}$ angular displacement.

\section{Validation 2}

The second validation experiment tested the effects of tilting on a model with a known center of rotation. A model was placed $180^{\circ}$ and $160^{\circ}$ away from the camera and then rotated three times in both clockwise and counterclockwise directions. WINanalyze software identified the center of rotation as the area defined by the grid markers that moved the least during rotation.

\section{Validation 3}

The third validation experiment tested whether placement of a model off-center inside the custom device influenced the determination of the known axial center of rotation. A model with a known center of rotation was created and potted in PVC (polyvinyl chloride) pipes. The upper bone had four holes drilled in it to change the known center of rotation. The model was placed inside the custom device so that the superior ring was attached to the model off-center, and a $7.05 \mathrm{Nm}$ rotational torque was applied. Five rotations of the model were recorded and tracked with WINanalyze at the four different known centers of rotation. WINanalyze software identified the center of rotation as the area defined by the five grid markers that moved the least during rotation.

\section{Validation 4}

The fourth validation experiment examined the effects of translation. A cadaveric spine was tested in the custom six-DOF device without any translation with a $120 \mathrm{Nm}$ torque. The spine was then translated $1.36 \mathrm{~mm}$ horizontally to the right and to the left, with a $120 \mathrm{Nm}$ rotational torque. In a third trial, the segment was translated $4.65 \mathrm{~mm}$ superolaterally, while the custom device applied a $120 \mathrm{Nm}$ rotational torque. Each experimental condition was repeated three times in both clockwise and counterclockwise directions. WINanalyze software identified the average apparent axial center of rotation as the area defined by the five grid markers that moved the least during rotation.

\section{Experiment}

After validating the method, we tested five fresh human cadaveric spines. The segments were harvested via disarticulation at their respective facet joints. All musculature was removed carefully, leaving ligamentous structures intact, including the anterior and posterior longitudinal ligaments, intraspinous ligaments, and the ligamentum flavum. The supraspinous ligaments superior to the spinous process of the superior segment and inferior to the spinous process of inferior segment were cut, as the superior and inferior facet joints were dissected and disarticulated.

The inferior vertebral body was potted in a PVC pipe, and the specimen was placed in the neutral position with the disc oriented horizontally, such that the plaster did not exceed the vertebral disc line. Each segment was rigidly fixed at its base, allowing for full range of motion of the superior segment. A plastic grid held in place with pins was placed on the superior vertebra to allow for marker placement. Regions of the spinal segment were defined based on marker rows. A PVC pipe ring was placed around the superior vertebra using fixation screws such that the vertebra could freely rotate and translate during force application. A metal ring was mounted onto the superior PVC pipe ring.

A $36.3 \mathrm{~kg}$ (80 lb) Dacron fishing wire, 3 meters in length, was wrapped around the metal ring and draped across four pulleys, with two pulleys directly horizontal to the metal ring and two superior pulleys attached to the Instron 4411 (Instron; Norwood, Massachusetts) machine. One set of pulleys rotated the specimen in the clockwise direction, while the other set rotated in the counterclockwise direction. Torques of 3.53, 7.05, 10.58, and $14.10 \mathrm{Nm}$ in both the clockwise and counterclockwise directions were applied to the specimens in random order. The custom six-DOF testing device applied the continual, pure, axial rotation force around a superior metal ring of the vertebra, allowing for movement along the $x$-, $y$-, and $z$-axes, along with rotation around the $x-, y$-, and $z$-axes, respectively.

Using WINanalyze software, we used markers to outline the vertebral body, spinal canal, facet joints, and the spinous process (Figure 2). Regions of the spinal segment were defined based on marker rows. Region 1 was defined as the posterior part of the vertebral body bordering the vertebral endplates, region 2 was defined as the anterior spinal canal, region 3 was defined as the middle spinal canal, region 4 was defined as the posterior spinal canal, and region 5 was defined as the area encompassing the facet joints and spinous process. The $x$ - and $y$-coordinates of each marker placed onto the specimen from the beginning to the end of each trial were found, and the 


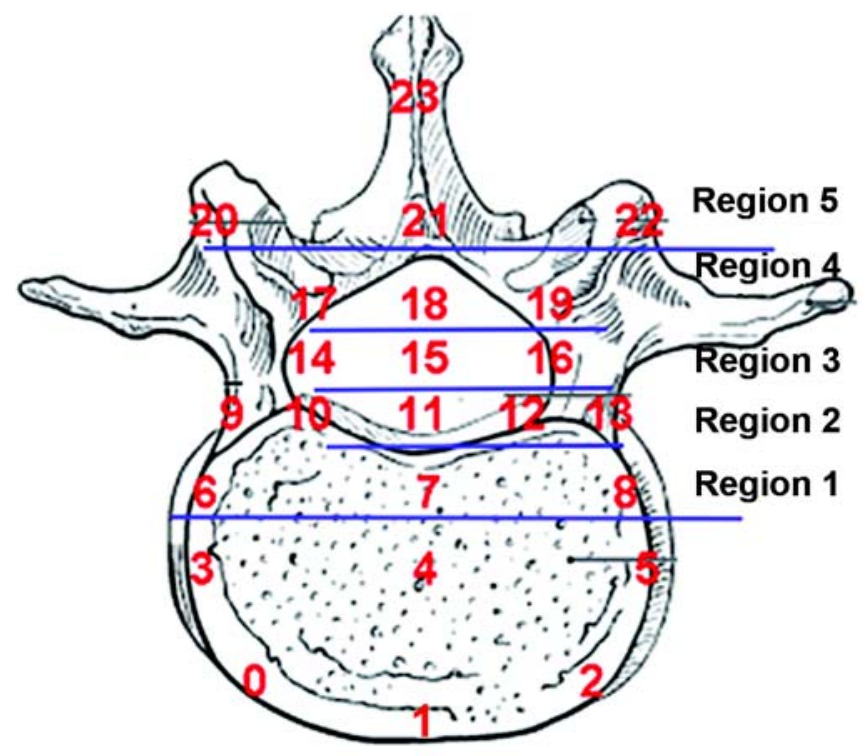

Figure 2.

Marker points established for data collection in sample lumbar spinal segment. Each row of markers is defined by a region, with region $1=$ posterior part of vertebral body bordering vertebral endplates, region $2=$ anterior spinal canal, region 3 = middle spinal canal, region 4 = posterior spinal canal, and region 5 = area encompassing facet joints and spinous process.

total distance that each marker moved was calculated. The five markers that moved the least distance were connected to define the axial center of rotation. The five markers that moved the least in the clockwise direction and the five that moved the least in the counterclockwise direction were calculated and drawn, and the area that overlapped between the clockwise and counterclockwise rotation experiments was used to determine the axial center of rotation. The marker angular displacement was calculated as the angle between a virtual line drawn between two points at the initial and final torque conditions. From the angular displacement data, the stiffness of the $\mathrm{L}$ specimens was also calculated. In total, seven L segments (three L4-L5, two L2-L3, and two L1-L2) and six T segments (T10-11, T8-9, T6-7, T4-5, T2-3, and cervical 7-T1) were tested.

\section{RESULTS}

\section{Validation 1}

Validation testing found that the grid marker placed on the known center of rotation moved an average of $0.004 \mathrm{~mm}$. The grid markers placed farther from the known center of rotation moved a greater distance. In order from least to greatest amount of distance from the known center of rotation, the grid markers moved 0.07 , $0.14,0.21,0.27$, and $0.34 \mathrm{~mm}$, respectively. A second set of grid markers placed in order from least to greatest distance from the known center of rotation moved 0.01, $0.19,0.29,0.38$, and $0.48 \mathrm{~mm}$, respectively.

\section{Validation 2}

The grid marker placed on the known center of rotation moved an average of $0.88 \mathrm{~mm}$ at $180^{\circ}$ and $0.21 \mathrm{~mm}$ at $160^{\circ}$ tilt. Additional grid marker points placed in order from least to greatest distance away from the known center of rotation at $180^{\circ}$ had a displacement of 3.35, 6.59, 10.20 , and $13.33 \mathrm{~mm}$. Another set of marker points at $180^{\circ}$ placed in order from least to greatest distance from the known center of rotation had a displacement of 4.79 , 8.09, 12.43, and $15.54 \mathrm{~mm}$. At a $160^{\circ}$ tilt, the first set of marker points had a displacement of 2.76, 7.51, 10.11, and $13.80 \mathrm{~mm}$, while the second set of grid makers displaced 2.23, 5.54, 10.02, and $10.86 \mathrm{~mm}$.

\section{Validation 3}

The five marker points positioned closest to the known center of rotation in the first trial had a displacement of 2.22, 6.03, 7.23, 7.48, and 8.06 mm. Markers positioned farther away from the known center of rotation had a displacement of 12.07, 12.27, 12.61, 12.76, 18.32, 18.46, 19.21, and $19.32 \mathrm{~mm}$. In the second trial, with a second known center of rotation, the five markers positioned closest to the known center of rotation had a displacement of 1.53, 1.88, 3.17, 3.57, and $5.65 \mathrm{~mm}$. Markers positioned further away from the known center of rotation had a displacement of 7.07, 7.29, 8.15, 8.78, 9.42, 10.36, and $13.65 \mathrm{~mm}$. In the third trial, with a third known center of rotation, the five markers positioned closest to the known center of rotation had a displacement of $0.80,1.00,1.67,4.45$, and $6.26 \mathrm{~mm}$. Markers positioned farther away from the known center of rotation had a displacement of 6.33, 6.73, 7.46, 9.56, 10.32, 12.58 , and $16.36 \mathrm{~mm}$. In the fourth trial, with a fourth known center of rotation, the five markers positioned closest to the known center of rotation had a displacement of 1.95, 2.69, 3.19, 4.82, and $5.56 \mathrm{~mm}$. Markers positioned farther away from the known center of rotation had a displacement of 5.74, 5.81, 8.56, 9.04, 9.26, $10.04,10.88$, and $20.40 \mathrm{~mm}$. In each trial, the five markers 
closest to the known center of rotation were able to define the known center of rotation.

\section{Validation 4}

The average apparent center of rotation was compared in three different testing conditions: no translation, $1.36 \mathrm{~mm}$ of horizontal translation to the right and to the left, and a $4.65 \mathrm{~mm}$ superolateral translation. In all three testing conditions, the apparent axial center of rotation was in region 1 (defined as the posterior part of the vertebral body bordering the vertebral endplates) and region 2 (defined as the anterior spinal canal).

\section{Experiment}

In the seven $\mathrm{L}$ spinal segments, rotations at 3.53, 7.05, 10.58, and $14.10 \mathrm{Nm}$ were averaged in the clockwise and counterclockwise directions, with the five points that moved the least forming an area in regions 1 and 2 corresponding to the posterior part of the vertebral body bordering the vertebral endplates and the anterior part of the spinal canal. One marker did not overlap in the clockwise and counterclockwise directions (Figure 3). The clockwise to counterclockwise average angular displacement ratio of the $\mathrm{L}$ spinal segments was 0.87 . The stiffness of the L segments was determined with an average stiffness of $2.18 \mathrm{Nm} /{ }^{\circ}$. In the six $\mathrm{T}$ segments, rotations at $3.53,7.05,10.58$, and $14.10 \mathrm{Nm}$ were averaged in the clockwise and counterclockwise directions, with the five points that moved the least forming an area in regions 1 and 2 corresponding to the posterior part of the vertebral body bordering the vertebral endplates and the anterior part of the spinal canal. All markers overlapped in the clockwise and counterclockwise directions (Figure 4). The clockwise to counterclockwise average angular displacement ratio of the T spinal segments was 0.97.

\section{DISCUSSION}

Developing a new method to determine the apparent axial center of rotation of the $\mathrm{L}$ spine allowed for complex motion to be broken down into individual components. The study began with developing a way to determine the apparent axial center of rotation, while maintaining as much natural spinal motion as possible via a custom sixDOF device. After developing a spine jig where pulleys applied torque on the superior vertebrae, we performed validation experiments in order to ensure that the design

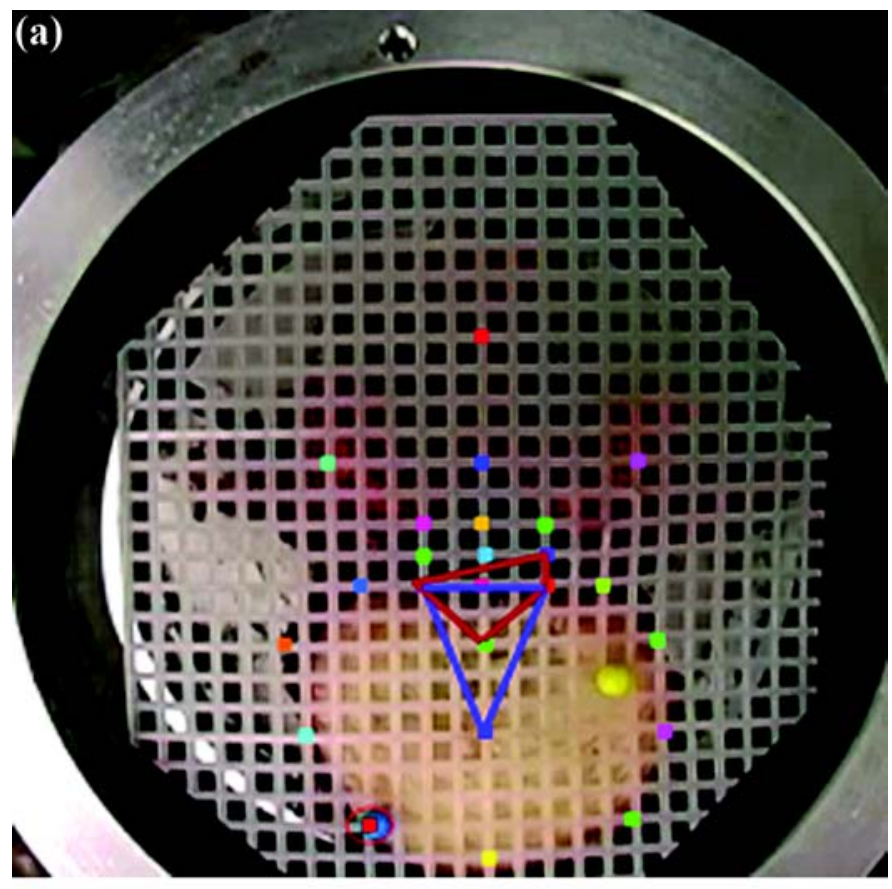

(b)

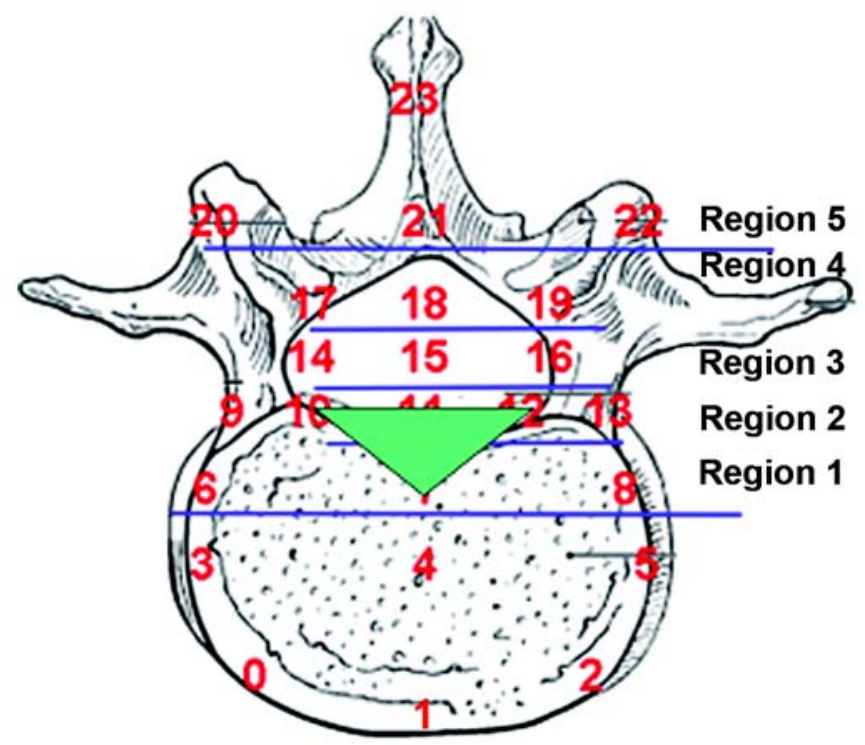

Figure 3.

(a) Apparent axial center of rotation in clockwise (red) and counterclockwise (blue) directions in lumbar (L) segments. Five markers that moved least in clockwise direction and five that moved least in counterclockwise direction were calculated and drawn, and area that overlapped between clockwise and counterclockwise rotation experiments was used to determine axial center of rotation. (b) Overlapping area determined to be center of rotation shown in green. Across all L specimens $(n=7)$, average apparent axial center of rotation fell within regions 1 and 2 . 

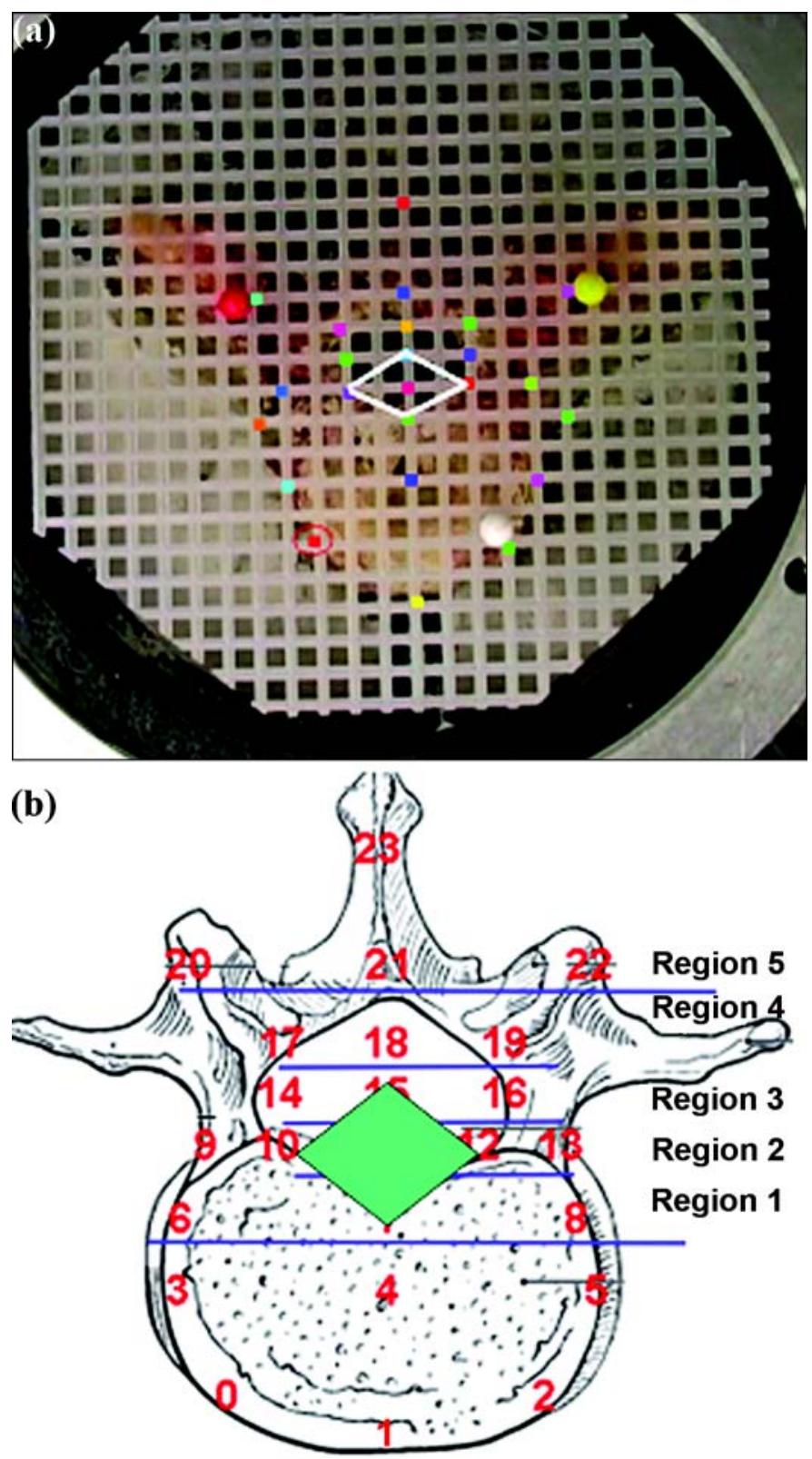

Figure 4.

(a) Apparent axial center of rotation in thoracic (T) segments (white). All five points overlapped in clockwise and counterclockwise directions. (b) Across all T specimens $(n=6)$, apparent axial center of rotation fell within regions 1,2 , and 3 (green).

was not flawed in determining the apparent axial center of rotation. The first validation experiment revealed that the custom device and WINanalyze software accurately determined the axial center of rotation in a model with a known axial center of rotation. Moreover, this validation model demonstrated that as the markers were placed far- ther from the known center of rotation, they moved a greater distance. The second validation experiment revealed that tilt did not influence the determination of the axial center of rotation. The tilt did, however, decrease the absolute values of the marker motion, indicating that the apparent axial center of rotation was decreased as the model was angled toward the camera. Absolute values were different in the model when it was flat compared with when it was tilted, but the known center of rotation remained the same. In the third validation experiment, placement of the model off-center in relation to the device placed the five markers that moved the least around the known center of rotation. In each of the four different known centers of rotation, the five markers that moved the least were able to define the known center of rotation. In the fourth validation experiment, translating the model while applying a rotational force had no effect on determining the apparent axial center of rotation. Therefore, validation testing verified that the custom device and WINanalyze software accurately determined the apparent axial center of rotation in a model with a known axial center of rotation. Tilting the segment, placing the segment off-center in relation to the device, and translating the segment had no effect on determining the apparent axial center of rotation.

The center of axial rotation of the spine in previous studies has shown conflicting results, placing the center anywhere from posterior to the spinous process to anterior to the vertebral body to as far lateral as the tip of the transverse process. Methods for determining the center of rotation of the spine vary from radiographic analysis to cadaveric measurements to geometric calculations. The difficulty in accurately determining the spinal center of rotation is highlighted in studies by Gregersen and Lucas, Nash and Moe, Panjabi et al., Kotani et al., and Molnár et al. $[8,10,12-14]$.

Gregersen and Lucas inserted pins during in vivo measurement of spinal motion in human subjects via a transducer [8]. Their experiments confirmed their geometric assumption that the center of rotation of any $\mathrm{T}$ or $\mathrm{L}$ vertebrae can be determined by the intersection of two lines passing through the centers of the surfaces of the articular facets and drawn perpendicular to the plane of these articular surfaces. Nevertheless, authors have argued that in vivo experiments are flawed secondary to unknown loads being applied to the spine, which lead to difficulty in accurate measurements [16-18].

Nash and Moe used radiographic analysis to determine vertebral rotation in the $\mathrm{T}$ and $\mathrm{L}$ spine [10]. Using 
rotation of the spinous process combined with pedicle shadow displacement, the authors developed a grading system (0-4) that attempted to demonstrate differences in the center of rotation at each vertebra. They concluded that the center of rotation was near the line of the posterior longitudinal ligament. However, radiographic analysis and a devised grading system of each vertebra cannot accurately determine the axial center of rotation in the spine. Panjabi et al. developed a spine jig to determine the $\mathrm{T}$ center of rotation through the application of flexion and extension forces [12]. They potted a third of the inferior vertebrae and placed a displacement jig on the superior vertebrae, applying direct force onto the superior vertebra. Their experiment found significant variability in determination of the center of rotation, and although they used an experimental jig, they were not able to accurately determine baseline values for normal spinal behavior. Kotani et al. attempted to determine the center of rotation with lateral plain films in flexion and extension by superimposing the images and comparing vertebral position [13]. After applying a moment of $8 \mathrm{Nm}$, they demonstrated that the center of rotation locations were highly variable.

Molnár et al.'s recent studies attempted to combine various methods of determining the center of rotation and apply them to the $\mathrm{T}$ spine [14]. Their experimental approach in the $\mathrm{T}$ spine included geometric correlation, volume change, and cadaveric spine testing. Despite variability in their studies, they summarized their results and concluded that the center of rotation was most likely near the anterior portion of the spinal canal. However, the cadaveric model in their study fixated both the upper and lower vertebrae in the test specimens, not allowing for full range of motion of the upper vertebrae.

The current study presents a new method of approximating the apparent center of rotation, allowing a full six DOF of movement in the upper vertebrae, using a video digitizing measurement technique. The findings reveal that the apparent axial center of rotation in the $\mathrm{L}$ spine for an individual spinal unit is located in regions 1 and 2, corresponding to the posterior border of the vertebral endplates and the anterior part of the spinal canal, with the $\mathrm{L}$ segments demonstrating an apparent axial center of rotation slightly anterior to the $\mathrm{T}$ segments. The area encompassing the axial center of rotation in the $\mathrm{T}$ segments is more posterior than the $\mathrm{L}$ spinal segments, with markers in both the counterclockwise and clockwise directions overlapping. The L segments demonstrated a marker difference in the counterclockwise and clockwise directions, but the overlapping area remained at the posterior border of the vertebral endplates and the anterior part of the spinal canal. This can be attributed to the fact that the L segments did not have equal angular displacement at the same forces. Under the influence of a standard force, the angular displacement varied in our experiment in opposing directions, with more displacement for a given force occurring in the counterclockwise direction than in the clockwise direction in the $\mathrm{L}$ spinal segments. This may result from intrinsic differences within each specimen or from unilateral disc compression or osteophytes on the intervertebral disc. These differences could potentially cause the axial center of rotation to deviate from the midline or shift slightly to the anterior or posterior, depending on where the osteophytic bone regions limit axial rotation.

One limitation of this study is the that center of rotation for axial rotation was evaluated without accounting for compression and shear forces; however, our data from the L spinal segments do correlate with previous in vivo data placing the $\mathrm{L}$ center of rotation at the posterior portion of the intervertebral disc [19-20]. Moreover, this study also verifies Molnár et al.'s hypothesis on the T center of rotation [14]. The multiple experimental setups yielded varied results on the position of the $T$ rotational axis; yet their summary identified the most likely place of the rotational axis as the posterior border of the vertebral endplates and the anterior part of the spinal canal. Our study verifies their hypothesis using our custom six-DOF device, which allowed for superior vertebral motion and rotation in all directions.

\section{CONCLUSIONS}

Returning to the basics of segmental motion will allow for an accurate determination of the center of rotation. The determination of the center of axial rotation of the spine will affect the treatment of back injury, a leading cause of pain and disability for adults in this country, with more than $\$ 34$ billion spent annually on health costs [21]. Freeman and Davenport believe that the best way to treat back pain surgically is to preserve physiological motion in the segment, which would theoretically minimize or prevent adjacent level degeneration [22]. With a better understanding of the biomechanics and the prospect of an increasing percentage of elderly patients, the 
future of treatment for back pain and instability, particularly in the L segments, may need to be reexamined. Pathological or surgical interventions for the spine could also potentially be improved through a better understanding of the center of rotation in the spine, through treatments that attempt to preserve physiological motion. Additional studies are needed to determine the pure center of rotation in the spine by combining the results of bilateral bending, compression, and flexion-extension data. Moreover, the center of rotation of the entire intact spine could provide clues into the coupled motion that occurs during physiological movement.

\section{ACKNOWLEDGMENTS}

\author{
Author Contributions: \\ Study concept and design: C. D. Rosen, T. Q. Lee. \\ Acquisition of data: S. P. Samagh, K. Otarodifard, M. Kornswiet, \\ G. Palmer. \\ Analysis and interpretation of data: S. P. Samagh, K. Otarodifard, \\ M. Kornswiet, G. Palmer. \\ Drafting of manuscript: S. P. Samagh, C. D. Rosen, T. Q. Lee. \\ Critical revision of manuscript for important intellectual content: \\ T. Q. Lee. \\ Study supervision: C. D. Rosen, T. Q. Lee. \\ Obtained funding: T. Q. Lee. \\ Financial Disclosure: The authors have declared that no competing \\ interests exist.
}

Funding/Support: This material is the result of work supported with resources and the use of facilities at the Department of Veterans Affairs Rehabilitation Research and Development Service, Long Beach Healthcare System, Long Beach, California.

\section{REFERENCES}

1. Cossette JW, Farfan HF, Robertson GH, Wells RV. The instantaneous center of rotation of the third lumbar intervertebral joint. J Biomech. 1971;4(2):149-53.

[PMID: 5119409] DOI:10.1016/0021-9290(71)90025-X

2. Lindahl O. Resection of vertebral transverse processes in idiopathic scoliosis. Acta Orthop Scand. 1966;37(4):342-47. [PMID: 5963290]

DOI:10.3109/17453676608989424

3. Oxland TR, Hoffer Z, Nydegger T, Rathonyi GC, Nolte LP. A comparative biomechanical investigation of anterior lumbar interbody cages: Central and bilateral approaches. J Bone Joint Surg Am. 2000;82(3)383-93. [PMID: 10724230]

4. Panjabi M, Henderson G, Abjornson C, Yue J. Multidirectional testing of one- and two-level ProDisc-L versus simu- lated fusions. Spine (Phila Pa 1976). 2007;32(12):1311-19. [PMID: 17515820$]$

5. Roaf R. The basic anatomy of scoliosis. J Bone Joint Surg Br. 1966;48(4):786-92. [PMID: 5953815]

6. Valdevit A, Errico TJ. Design and evaluation of the FlexiCore metal-on-metal intervertebral disc prosthesis. Spine J. 2004;4(6 Suppl):276S-88S. [PMID: 15541677] DOI:10.1016/j.spinee.2004.07.016

7. Wagner H, Anders Ch, Puta Ch, Petrovitch A, Mörl F, Schilling N, Witte H, Blickhan R. Musculoskeletal support of lumbar spine stability. Pathophysiology. 2005;12(4): 257-65. [PMID: 16239098] DOI:10.1016/j.pathophys.2005.09.007

8. Gregersen GG, Lucas DB. An in vivo study of the axial rotation of the human thoracolumbar spine. J Bone Joint Surg Am. 1967;49(2):247-62. [PMID: 6018729]

9. Rockwell H, Evans FG, Pheasant HC. The comparative morphology of the vertebrae spinal column. Its form as related to function. J Morphol. 1938;63(1):87-117. DOI:10.1002/jmor.1050630105

10. Nash CL Jr, Moe JH. A study of vertebral rotation. J Bone Joint Surg Am. 1969;51(2):223-29. [PMID: 5767315]

11. Roaf R. Rotation movements of the spine with special reference to scoliosis. J Bone Joint Surg Br. 1958;40-B(2): 312-32. [PMID: 13539116]

12. Panjabi MM, Krag MH, Dimnet JC, Walter SD, Brand RA. Thoracic spine centers of rotation in the sagittal plane. J Orthop Res. 1984;1(4):387-94. [PMID: 6491788] DOI:10.1002/jor.1100010407

13. Kotani Y, Cunningham BW, Abumi K, Dmitriev AE, Hu N, Ito M, Shikinami Y, McAfee PC, Minami A. Multidirectional flexibility analysis of anterior and posterior lumbar artificial disc reconstruction: In vitro human cadaveric spine model. Eur Spine J. 2006;15(10):1511-20.

[PMID: 16552532]

DOI:10.1007/s00586-006-0086-Z

14. Molnár S, Manó S, Kiss L, Csernátony Z. Ex vivo and in vitro determination of the axial rotational axis of the human thoracic spine. Spine (Phila Pa 1976). 2006;31(26):E984-91. [PMID: 17172989]

15. Knutsson F. The instability associated with disc degeneration in the lumbar spine. Acta Radiol. 1944;25:593-609. DOI:10.3109/00016924409136488

16. Busscher I, Van Dieën JH, Kingma I, Van der Veen AJ, Verkerke GJ, Veldhuizen AG. Biomechanical characteristics of different regions of the human spine: An in vitro study on multilevel spinal segments. Spine (Phila Pa 1976). 2009;34(26):2858-64. [PMID: 20010393]

17. Panjabi MM, Oxland TR, Yamamoto I, Crisco JJ. Mechanical behavior of the human lumbar and lumbosacral spine as shown by three-dimensional load-displacement curves. 
J Bone Joint Surg Am. 1994;76(3):413-24. [PMID: 8126047]

18. Sizer PS Jr, Brismée JM, Cook C. Coupling behavior of the thoracic spine: A systematic review of the literature. J Manip Physiol Ther. 2007;30(5):390-99. [PMID: 17574958] DOI:10.1016/j.jmpt.2007.04.009

19. Rousseau MA, Bradford DS, Hadi TM, Pedersen KL, Lotz JC. The instant axis of rotation influences facet forces at L5/S1 during flexion/extension and lateral bending. Eur Spine J. 2006;15(3):299-307. [PMID: 16175392] DOI:10.1007/s00586-005-0935-1

20. Xia Q, Wang S, Kozanek M, Passias P, Wood K, Li G. In-vivo motion characteristics of lumbar vertebrae in sagittal and transverse planes. J Biomech. 2010;43(10):1905-9.

[PMID: 20381051]

DOI:10.1016/j.jbiomech.2010.03.023

21. Frymoyer JW, Durrett CL. The economics of spinal disorders. In: Frymoyer JW, ed. The adult spine: Principles and practice. Philadelphia (PA): Lippincott-Raven; 1997. p. 143-50.
22. Freeman BJ, Davenport J. Total disc replacement in the lumbar spine: A systematic review of the literature. Eur Spine J. 2006;15 Suppl 3:S439-47. [PMID: 16862432] DOI:10.1007/s00586-006-0186-9

Submitted for publication September 2, 2010. Accepted in revised form November 22, 2010.

This article and any supplementary material should be cited as follows:

Samagh SP, Rosen CD, Otarodifard K, Kornswiet M, Palmer G, Lee TQ. New method for determining apparent axial center of rotation of lumbar and thoracic spine segments. J Rehabil Res Dev. 2011;48(5):587-96.

DOI:10.1682/JRRD.2010.09.0168

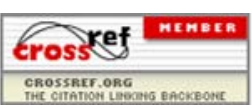


